\title{
The theorem of Morera in several variables.
}

Memoria di Salomon Bochen (a Princeton, N. J., U. S. A.).

Sunto. 亡̀ dato dal seguente capoverso.

The elassical theorem of Morera has been generalized from one to several variables by VoLTerRA and Severi ( ${ }^{1}$ ), and SEveri's proof is very brief and elegant. We will re-prove this theorem (our Theorem 7 ) by a chain of reasoning which, to the contrary, will be lengthy and elaborate, but our actual contributions are the intermediary results and the method of proof, rather than the final proposition itself.

In the Euclidean $E_{2 k}$ of $k$ complex variables $z^{j}=x^{j}+i y^{j}, j=1, \ldots, k$, we will distinguish between functions which have power series in $z^{j}-z_{0}^{j}$ locally; and such ones which have power series in $x^{j}-x_{0}{ }^{j}, y^{j}-y_{0}{ }^{j}$, or, what is the same, in $z^{j}-z_{0}^{j}, z^{j}-z_{0}^{j}$ locally. The first ones we will call "holomorphic"; and the second ones we will term "real-analytic», even if their values themselves will be complex numbers, as a rule. Also, if a function has power series in $\overline{z^{j}}-\overline{z_{0}^{j}}$ only, we will call it «anti-holomorphic».

\section{General Cycles.}

In the complex $(x, y)$-plane we formally introduce the variables

$$
z=x+i y, \quad \vec{z}=x-i y
$$

and we denote an arbitrary function $F(x, y)$ by $f(z, \bar{z})$ in accordance with a well-known symbolism ( $\left.{ }^{2}\right)$. The classical theorem of Morera then states that if such a function is defined and continuous in a domain $D$ and if we then have

$$
\int_{B_{1}} f d z=0
$$

(1) F. SEVERT, Sur une propriété fondamentale des fonctions analytiques de plusieurs variables complexes, Comptes Rendns, Paris, 192, (1931).

(2) Compare, for instance, S. Bochner and W. T. Martix, Several complex variables, (1948), p. 37 . 
over every 1-cycle $B_{i}$, then $f$ is holomorphic:

$$
\frac{\partial f}{\partial z}=0
$$

and, of course, dually, if we have

$$
\int_{B_{1}} f d \bar{z}=0
$$

then $f$ is anti-holomorphic

$$
\frac{\partial f}{\partial z}=0
$$
follows.

Now, perhaps the most natural generalization of this theorem is as

Theorem $1\left({ }^{3}\right)$. - In the space $E_{z h}$ of the $k$ complex variables $z^{x}=x^{x}+i y^{x}$. $\alpha=1, \ldots, k$, if

$$
f=f\left(z^{1}, \ldots, z^{k}, \overline{z^{1}}, \ldots, \bar{z}^{k}\right)
$$

is continuous in a domain $D$, and if we then have

$$
\int_{B_{p+q}^{\prime}} f d z^{\alpha_{1}} \ldots d z^{z_{p}} d \bar{z}^{\beta_{1}} \ldots d \overline{z^{\beta_{q}}}=0
$$

over every $(p+q)$-dimensional cycle $B_{p+q}$, where

$$
1 \leq \alpha_{1}<\alpha_{2}<\ldots<\alpha_{p} \leq k, \quad 1 \leq \beta_{1}<\beta_{2}<\ldots<\beta_{q} \leq k
$$

are fixed indices with

$$
0 \leq p, \quad 0 \leq q, \quad 1 \leq p+q \leq 2 k-1,
$$

then $f$ is holomorphic in those $\mathrm{z}^{\beta}$, which are different from the given ones, and anti-holomorphic in those $z^{x}$ which are different from the given ones; that is

$$
\begin{aligned}
& \frac{\partial f}{\partial z^{\alpha}}=0, \quad \alpha \neq \alpha_{1}, \ldots, \alpha_{p} \\
& \frac{\partial f}{\partial z^{\beta}}=0, \quad \beta \neq \beta_{1}, \ldots, \beta_{q} .
\end{aligned}
$$

(3) For the propositions of which Theorem 1 is the converse see B. SEGRE, Sull'esten. sione della formula integrale di Cauchy e sui residui degli integrali $n$-pli nella teoria delle funzioni di "variabili complesse, "Atti I Congresso U. M. I.", Bologna, (1937), and E. MartineLLi, kormule Integrali e topologia nella teoria delle funzioni di pin variabili com. plesse, "Acta Pontif Acad. Scient.", IX, p. 235.250. 
Proof. We first note that, for instance:

$$
\int_{B_{k}} f d z^{\wedge} \ldots d z^{k}=0
$$

implies that $f$ is holomorphic in all $z^{x}$, that is

$$
\frac{\partial f}{\partial \bar{z}^{\beta}}=0, \quad \beta=1, \ldots, k
$$

and that, for instance, for $k \geq 2$,

$$
\int_{B_{2}} f d z^{1} d \overline{z^{1}}=0
$$

implies that $f$ is «arbitrary continuous in $z^{1}, \vec{z}^{1}$ : that is in $x^{1}, y^{\prime}$ but that it is constant in all the other variables, that is

$$
\frac{\partial f}{\partial z \gamma}=0=\frac{\partial f}{\partial z^{Y}}, \quad \gamma=2, \ldots, k
$$

and hence

$$
\frac{\partial F}{\partial x^{\gamma}}=0=\frac{\partial F}{\partial y^{\gamma}}=0, \quad \gamma=2, \ldots, k .
$$

For the proof we note that if $f$ has also continuons first derivatives in all $x$, $y$, then the theorem is a direct consequence of STokes' theorem. In fact, if $B_{p+q}$ bounds a chain $B_{p+q+1}$ then (2) implies

$$
\int_{B_{p+q+1}} d f d z^{x_{1}} \ldots d z^{\alpha_{p}} d \overline{z^{\beta_{1}}} \ldots d \bar{z}_{\beta_{q}}^{\beta_{1}}=0
$$

and since $B_{p+q+1}$ can be an arbitrary simplex, this implies

$$
d f d z^{\alpha_{1}} \ldots d z^{\alpha^{\alpha}} d \bar{z}^{\beta_{1}} \ldots d \bar{z}^{\beta_{q}}=0
$$

where

$$
d f=\frac{\partial f}{\partial z^{1}} d z^{1}+\ldots+\frac{\partial f}{\partial z^{k}} d z^{k}+\frac{\partial f}{\partial \bar{z}_{1}} d \bar{z}^{1}+\ldots+\frac{\partial f}{\partial \bar{z}_{k}} d \bar{z}^{k}=0 .
$$

However, for fixed $\alpha_{p}, \rho=1, \ldots, p ; \beta_{\sigma}, \sigma=1, \ldots, q$; and variable $\alpha \neq \alpha_{p}$, $\beta \neq \beta_{\text {, }}$ the $(k-p)+(k-q)$ monomials

$$
d z^{x} d z^{x_{1}} \ldots d z^{x_{p}} d \bar{z}^{\beta_{1}} \ldots d \bar{z}_{\beta_{q}}, \quad d \bar{z}^{\beta} d z^{\alpha} \ldots d z^{\alpha_{p}} d \bar{z}^{\beta_{1}} \ldots d z^{q_{q}}
$$

are all linearly independent, and this implies relation (5), (6). 
Next, if it is only known that $f$ is continuous, we will have to apply an approximation process and the underlying approximation theorem is as follows $\left({ }^{4}\right)$.

In (real) Euclidean $E_{n}:\left(t^{1}, \ldots, t^{n}\right)$ it is possible to construct a sequence of functions $\left\{\varphi_{\mu}\left(t^{1}, \ldots, t^{n}\right)\right\}, \mu=1,2,3, \ldots$, having the following properties.

(i) Each $\varphi_{\mu}(t)$ is defined in all of $E_{n}$ but vanishes outside a sphere

$$
K_{\mu}:\left(t^{1}\right)^{2}+\ldots+\left(t^{n}\right)^{3} \leq \delta_{\mu}^{2},
$$

where

$$
\lim _{\mu \rightarrow \infty} \delta_{\mu}=0 \text {. }
$$

(ii) Each $\varphi_{\mu}(t) \varepsilon C^{\infty}$ in $E_{n}$, that is, it has continuous partial derivatives of every mixed order.

(iii) $\varphi_{\mu}(t) \geq 0$, and

$(i v)$

$$
\int_{E_{E^{\prime}}} \varphi_{\mu}(t) d v_{t}=\int_{\overleftarrow{K}_{\mu}} \varphi_{\mu}(t) d v_{t}=1 .
$$

Now, if $F\left(x^{1}, \ldots, x^{n}\right)$ is defined and contintous and also bounded in a domain $D$, then we supplement it by values 0 outside $D$, and we set up the expressions

$$
\begin{aligned}
F_{\mu}\left(x^{1}, \ldots, x^{n}\right) & =\int_{E_{n}} F\left(x^{1}+t^{1}, \ldots, x^{n}+t^{n}\right) \varphi_{\mu}\left(t^{1}, \ldots, t^{n}\right) d v_{t} \\
& =\int_{K_{\mu}} F\left(x^{1}+t^{1}, \ldots, x^{n}+t^{n}\right) \varphi_{\mu}\left(t^{1}, \ldots, t^{n}\right) d v_{t} .
\end{aligned}
$$

for $x=\left(x^{\nu}\right)$ in $D ; \mu=1,2, \ldots$. These expressions are approximating functions in the following sense $\left({ }^{5}\right)$.

Lemma 1. - (i) Each $F_{\mu}(x) \in C^{\infty}$ in $E_{n}$, and if $D^{\prime} \subset D$ is a subdomain whose closure is likewise contained in $D, \bar{D}^{\prime} \subset D$, then

uniformly in every $D^{\prime}$.

$$
\lim _{\mu \rightarrow \infty} F_{\mu}(x)=F(x)
$$

(ii) If, moreover, $F \in C^{r}$ in $D, r \geq 1$, then we also have

$$
\lim _{\mu \rightarrow \infty} \frac{\partial q_{1}+. .+q_{n} F_{\mu}}{\partial\left(x^{1}\right)^{q_{1}} \ldots \partial\left(x^{n}\right)^{q_{n}}}=\frac{\partial q_{1}+\ldots+q_{n} F}{\partial\left(x^{1}\right)^{q_{1}} \ldots \partial\left(x^{n}\right)^{q_{n}}}
$$

for $0 \leq q_{1}+\ldots+q_{n} \leq r$, again uniformly in $D^{\prime}$.

(4) S. Bochner and W. T. MARTIN, loc. cit, p. 109.13.

(5) Bochner and MartiN, loc. cit., p. 36 . 
Returning to our Theorem 1, if (2) holds for «all» cycles $B_{p+q}$, and if we pick a fixed such cycle, then it also holds for «translated》 cycles $B_{p+q}-(t)$ for small $|t|$. However in the relation (2), instead of translating the cycle, by $-t$, we can also translate the argument of the function by $+t$. That is, if we denote the function (1) for the moment by

$$
F\left(x^{1}, \ldots, x^{n}\right) \quad(n=2 k),
$$

then (2) also holds for the function

$$
F\left(x^{1}+t^{1}, \ldots, x^{n}+t^{n}\right)
$$

for sufficiently small $t$. For given $B_{p+q}$, we can then multiply the resulting relation by $\varphi_{\mu}(t)$ and integrate over $\bar{K}_{\mu}$, for $\mu$ sufficiently large, and thus, we obtain

$$
\int_{\dot{B}_{p+q}} f_{\mu}(z, \bar{z}) d z^{\alpha_{1}} \ldots d z^{\alpha_{p}} d \bar{z}^{\beta_{1}} \ldots d \bar{z}^{\beta_{q}}=0,
$$

for $\mu \geq \mu\left(B_{p+q}\right)$. But $f_{\mu} \varepsilon C^{1}$ and thas we have our conclusions

$$
\begin{aligned}
& \frac{\partial f_{\mu}}{\partial z^{\alpha}}=0, \quad \alpha \neq \alpha_{1}, \ldots, \alpha_{p} \\
& \frac{\partial f_{\mu}}{\partial z^{\beta}}=0, \quad \beta \neq \beta_{1}, \ldots, \beta_{q},
\end{aligned}
$$

for $\mu \geq \mu\left(D^{\prime}\right)$, for every subdomain $D^{\prime}$ as before. However, in $D^{\prime}, f_{\mu}$ converges uniformly towards $f$; and by WEIERSTRAss' theorem on the uniform limit of holomorphic functions, which also holds for anti-holomorphic functions. we now obtain the relations (5), (6) themselves, q. e. d.

\section{Rigidly-places products of one-cycles.}

In the $z^{2}$-plane, $j=1, \ldots, k$, we take a one-dimensional cycle $B_{1}{ }^{\prime}$ which bounds a two-chain $B_{2}{ }^{j}$ and we consider the products

$$
B_{2 k}=B_{2}^{1} \times B_{2}^{2} \times \ldots \times B_{2}{ }^{n}
$$

For a given domain $D$, if (10) is contained in $D$, then we will say that (11) is a rigidly-placed products of one-cycles.

A «repeated» application of Srokes' theorem gives formally

$$
\begin{aligned}
\int_{B_{k}} f d z^{1} \ldots d z^{k} & =\int_{B_{2 k}} \frac{\partial^{k} f}{\partial \overline{z^{1}} \ldots \overline{\partial z^{k}}} d \overline{z^{i}} d z^{i} d \overline{z^{2}} d z^{2} \ldots d \overline{z^{k}} d z^{k} \\
& =(2 i)^{k} \int_{\bar{B}_{2 k}} \frac{\partial^{k} f}{\partial \overline{z^{1}} \ldots \partial z^{k}} d V_{x, y}
\end{aligned}
$$


where

$$
d V_{x, y}=d x^{1} d y d x^{2} d y^{2} \ldots d x^{k} d y^{k}
$$

is the ordinary volume-element, and, again with the aid of lemma 1 , the following conclusion can be drawn.

THEOREM 2. - If $f \in C^{k}$, and

$$
\frac{\partial^{k} f}{\partial \bar{z}^{4} \ldots \partial z^{k}}=0
$$

then we have

$$
\int_{B_{k}} f d z^{1} \ldots d z^{k}=0
$$

for every rigidly-placed product (11).

Conversely, if (13) holds for all such cycles in $D$, and it $f \varepsilon C^{k}$, then $f$ satisfies the equation (12), and if $f \cdot \varepsilon C^{\circ}$, then $f$ is a uniform limit in $D^{\prime}$ of solutions of $(12)$

If a function (1) is real-analytic and if we expand it in a power-series of $z^{x}, z^{x}$ in the neighborhood of the origin, say, then it is a solution of $\{12\}$ if and only if the expansion has the form

$$
\sum_{j=1}^{k} \sum_{(n)} f_{n_{1} \ldots n_{j-1} n_{j-L_{1}} \ldots n_{z}}(z)\left(\bar{z}^{1}\right)^{n_{1}} \ldots\left(\overline{z^{k}}\right)^{n_{k}}
$$

in which the coefficients $f_{1 \prime}$ are holomorphic in their variables. But we do not know whether any solution of (12) must automatically be real-analytic; and thus no absolute assertion can be formulated.

3. Affinely-placed produets of one-eycles.

We will say that a $k$-cycle $B_{k}$ in $\left.I\right)$ is an affinely-placed product of one-cycles if there is a non-singular holomorphic transformation with constant coefficients

$$
z^{j}=\sum_{l=1}^{k} a_{j l} w^{l}+a_{j},
$$

such that the image of $B_{k}$ in the $n$-space is a rigidly-placed product of one-cycles as previously defined.

Theorem 3. - If a function has the form

$$
f=\sum_{0 \leq p_{1}+\ldots+p_{k} \leq k-1} a_{p_{1} \ldots p_{k}}(z)\left(\overline{z^{1}}\right)^{p_{1}} \ldots\left(\overline{z^{k}}\right)^{p_{k}}
$$

with holoworphic coefficients

$$
a_{p}(z)=a_{p_{1} \ldots p_{k}}\left(z^{1}, \ldots, z^{k}\right)
$$


then we obviously have

$$
\frac{\partial^{h} f}{\partial z^{\alpha_{1}} \ldots \partial z^{\alpha_{k}}}=0
$$

for all possible combinations

$$
1 \leq \alpha_{1} \leq k, \quad 1 \leq \alpha_{2} \leq k, \ldots, \quad 1 \leq \alpha_{k} \leq k,
$$

and we have

$$
\int_{B_{k}} f d z^{1} d z^{2} \ldots d z^{k}=0
$$

for every affinely placed $B_{k}$.

Proof. If we introduce the linear operators

$$
L_{j}=\sum_{l=1}^{k} \bar{a}_{l j} \frac{\partial}{\partial \bar{z}^{b}}
$$

with any constant coefficients $a_{l j}$ then (18) implies

$$
L_{1} L_{2} \ldots L_{k} f=0 \text {, }
$$

and this means that we have property (12) even after any affine transformation (15). And the conclusion now follows from Theorem 2.

THEOREM 4. - If $f$ continuous in $D_{1}$ and if we have

$$
\int_{B_{k}} f d z^{1} \ldots d z^{k}=0
$$

for every affinely-placed $B_{k}$, then $f$ is real-analytic, and we have (18), and it can be represented as an expression (16).

Proof. Assume first that it is already known that $f$ is real-analytic. It is then in particular $C^{k}$, and (21) implies by Theorem 2 that we have $\left(20^{\prime}\right)$ for all possible operators (20), and hence (18). But $f$ being also real analytic, we can expand it in the neighborhood of every point $z_{0}{ }^{\alpha}$ in a power-series in the quanties $z^{\alpha}-z_{0}^{\alpha}, \bar{z}^{x}-\bar{z}_{0}^{\alpha}$, and due to (18) the expansion must be of the form

$$
\underset{0 \leq p_{1}+\ldots+p_{k} \leq k-1}{\Sigma} a_{p}\left(z ; z_{0}\right)\left(\overline{z^{1}}-\overline{z_{0}^{1}}\right)^{p_{1}} \ldots\left(\overline{z^{k}}-\bar{z}_{0}^{k}\right) p_{k}
$$

where $a_{p}\left(z ; z_{0}\right)$ is holomorphic in the $z^{z}$ in the neighborhood of the given point $z_{0} x$. Now, (22) is a polynomial of degree $\leq k-1$ in the $\bar{z}^{x}-\bar{z}_{0}^{x}$, and as such it is also a polynomial in the $\bar{z}^{x}$ themselves:

$$
\sum_{0 \leq p_{1}+\ldots+p_{k} \leq k-1}^{\sum} b_{p}\left(z ; z_{0}\right)\left(\overline{z_{1}^{1}}\right)^{p_{1}} \ldots\left(\overline{z^{k}}\right)^{p_{k}}
$$


with ceefficients $b_{p}\left(z ; z_{0}\right)$ which are holomorphic in the $z^{x}$, in a neighborhood of $z_{0}{ }^{\alpha}$. But by a known uniqueness theorem these coefficients must be identical with one another in the intersection of two such neighborhoods, and hence there is a representation (16) jointly for the entire domain $D$.

But now we must eliminate the assumption that $f$ is real-analytic, and we will perform this elimination in the following two steps, where the second step will again be based on our process of approximation as ensuing from lemma 1.

Step 1. If $f \in C^{k+t}$ and (18) holds for all combinations (19), then $f$ is real-analytic.

Step 2. If we are given a sequence of function $f_{\mu}$ in $D$, if each $f_{\mu}$ has a representation (16) with holomorphic coefficients (17) and if the sequence is uniformly convergent,

$$
\lim _{\mu \rightarrow \infty} f_{\mu}=f
$$

then $f$ itsel can likewise be represented in the form (16).

Now Step 1 obviously follows from the following more general theorem.

TheоReм 5. - If for some $l \geq 1, l \gtreqless k$, we are given a function $f \varepsilon 0^{l+1}$ and if the expressions

$$
g_{x_{1} \ldots \alpha_{l}}(z, \bar{z})=\frac{\partial^{l} f}{\partial z^{\alpha_{1}} \ldots \partial z^{\alpha_{l}}}
$$

for all possible combinations $\alpha_{1}, \ldots, \alpha_{l}$ are real-analytic (for instance. if they are all 0 ), then the function $f$ itself is real-analytic.

Proof. We will need the following lemma.

LEMMA 2. - If we are given formal power series

$$
g_{\alpha}(t)=\sum_{p_{j} \geqslant 0} a_{\alpha p_{1} \ldots p_{k}} t_{t}^{p_{1}} \ldots t_{k} p_{k}, \alpha=1, \ldots, k,
$$

in symbols $t_{1}, \ldots, t_{k}$ with coefficients from an arbitrary vector field over rational numbers. and if the integrabiliiy conditions

$$
\frac{\partial g_{\alpha}}{\partial t_{\beta}}=\frac{\partial g_{\beta}}{\partial t_{\alpha}}
$$

are fulfilled formally, then the series without constant term

with the coefficients

is such that we have

$$
G(t)=\sum_{p_{1}+\ldots+p_{k}>0} a_{p_{1} \ldots p_{k}} t_{1}^{p_{1}} \ldots t_{k} p_{k}
$$

$$
a_{p_{1} \ldots p_{k}}=\frac{\Sigma a_{\alpha p_{1} \ldots\left(p_{\alpha}-1\right) \ldots p_{k}}}{p_{1}+\ldots+p_{k}}
$$

$$
\frac{\partial G}{\partial t_{\alpha}}=g_{\alpha}(t), \quad(\alpha=1, \ldots, k) .
$$


Proof of Lemma $2{ }^{6}$ ). If (26) is to satisfy (28) then on putting

$$
H\left(\lambda_{1}, \ldots, \lambda_{k} ; s\right)=G\left(\lambda_{1} s, \ldots, \lambda_{k} s\right)
$$

we must have

$$
\begin{aligned}
\frac{\partial H}{\partial s} & =\sum_{\alpha} \frac{\partial G}{\partial t_{\alpha}} \lambda_{\alpha}=\sum_{\alpha} g_{\alpha} \lambda_{\alpha} \\
& =\sum_{p_{1}+\ldots+p_{k}>0} \frac{1}{s} \cdot\left(p_{1}+\ldots+p_{k}\right) a_{p_{1} \ldots p_{k}}\left(\lambda_{1} s\right)^{p_{1}} \ldots\left(\lambda_{k} s\right)^{p_{k}} \\
& =\underset{p, \alpha}{\Sigma} \lambda_{\alpha} a_{x p_{1}}, \ldots, p_{k}\left(\lambda_{1} s\right)^{p_{1}} \ldots\left(\lambda_{k} s\right)^{p_{k}}
\end{aligned}
$$

so that (27) is the only possible choice of coefficients to that purpose. Conversely, if we now set

$$
w_{\alpha}(\lambda ; s)=\frac{\partial H}{\partial \lambda_{\alpha}}-s h_{\alpha}
$$

where

$$
h_{\alpha}(\lambda ; s)=g_{\alpha}\left(\lambda_{1} s, \ldots, \lambda_{k} s\right)
$$

then we obtain

$$
\frac{\partial w_{\alpha}}{\partial s}=\frac{\partial}{\partial \lambda_{\alpha}}\left(\frac{\partial H}{\partial s}\right)-h_{\alpha}-s \Sigma_{\beta} \frac{\partial g_{\alpha}}{\partial t_{\beta}} \lambda_{\beta}
$$

and by (30) this is

$$
h_{z}+s \sum_{\beta} \lambda_{\beta} \frac{\partial g_{P}}{\partial t_{\alpha}}-h_{\alpha}-s \sum_{\beta} \frac{\partial g_{\alpha}}{\partial t_{\beta}} \lambda_{\beta}=0,
$$

by (25). Therefore, if (25) holds, then $w_{a}(\lambda ; s)$ must be independent of the formal variable $s$. But $w_{\alpha}(\lambda ; 0)=0$, and therefore

$$
\frac{\partial H}{\partial \lambda_{\alpha}}=s h_{\alpha}
$$

or what is the same

$$
\frac{\partial G}{\partial t_{x}}=g_{\alpha}
$$

as claimed.

Turning now to Theorem 5, it suffices to prove the theorem in the neighborhood of a point, and we will prove it by induction on $l$. For $l=1$, we have $f \varepsilon C^{2}$, and $(23)$ is

$$
g_{\alpha}(z, \bar{z})=\frac{\partial f}{\partial \bar{z}^{\alpha}}, \quad(\alpha=1, \ldots, k)
$$

(6) Compare our paper: Formal Lie groups, "Annals of Math. », 47, (1946), 192.201. 
Differentiating once more we obtain

$$
\frac{\partial g_{\alpha}}{\partial \bar{z}^{\beta}}=\frac{\partial g_{\beta}}{\partial \bar{z}^{z}}
$$

and by lemma 2 there exists locally a real-analytic function $G(z) \bar{z})$ such tha

$$
\frac{\partial G}{\partial \bar{z}^{\alpha}}=g_{x}(z, \bar{z}) \text {. }
$$

For the difference $\varphi=t-G$ we obtain

$$
\frac{\partial \varphi}{\partial \bar{z}^{\alpha}}=0
$$

thus $\varphi$ is holomorphic, and in particular real-analytic, and $f=\varphi+G$ is likewise so.

For $l>1$ if we put

$$
h=\frac{\partial^{l-1} f}{\partial \bar{z}^{\alpha_{1}} \ldots \partial \bar{z}^{\alpha_{l-1}}}
$$

for any fixed combination $\alpha_{1}, \ldots, \alpha_{l-1}$, then by assumption of theorem 5

$$
\frac{\partial h}{\partial \overline{z^{\alpha}}} \equiv g_{\alpha_{1}, \ldots \alpha_{l-1} \alpha}, \quad(\alpha=1, \ldots, k)
$$

is real-analytic, and by what we have just proven, the function $h$ is real-analytic itself. Therefore the expressions

$$
h_{\alpha_{1} \ldots \alpha_{l-1}}=\frac{\partial^{l-f}}{\partial \bar{z}^{x_{1}} \ldots \overline{\partial \bar{z}^{x_{l-1}}}}
$$

are real-analytic and if the theorem was known to hold now for $l-1$, then $f$ is real-analytic, and thus theorem holds for $l$ itself, as claimed.

We will make a remark to Theorem 5 for its own sake.

THeOREM 6. - In Theorem 5, instead of assuming $f \in C^{t+1}$, it is also sufficient to assume $f \in C^{l}$ and to add the explicit assumption that the integrability condition

$$
\frac{\partial g_{\alpha_{1} \ldots \alpha_{l-1_{1}}}}{\partial \bar{z}^{\beta}}=\frac{\partial g_{\alpha_{1} \ldots x_{l-1} \beta}}{\partial \bar{z}^{\alpha}}
$$

shall be valid.

Now, (31) is certainly valid if $g_{\alpha_{1} \ldots \alpha_{l-1} \alpha_{l}}=0$, as is especially the case for equations (18) with $l=k$, so that in step 1 it would have been sufficient to assume $f \varepsilon C^{k}$ instead of $f \varepsilon C^{k+1}$ B't for our context itself this is of no difference. 
Next, for the proof of step 2, it again suffices to deal with a neighborhood of a point, and if we expand each $a_{p}(z)$ in a power series, then it is not hard to see that the following lemma, of some interest in itself, is sufficient for the purpose. We will again in this lemma, as in lemma 2, place all indices as subscripts, instead of as superscripts.

LEMMa 3. - In the domain

$$
0 \leq\left|z_{j}\right| \leq 2,
$$

$$
(j=1, \ldots, k)
$$

we consider the absolutely convergent expansion

in which

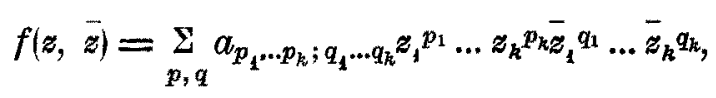

$$
0 \leq p_{j}<\infty, \quad 0 \leq q_{j} \leq l
$$

for some finite $l$, and we assume that we have

$$
|f(z, \bar{z})|<1
$$

for all points in (32).

There exist a constant $L=L(k, l)$ such that

$$
\sum_{p, q}\left|a_{p, q}\right|\left|z_{1}\right|^{p_{1}+q_{1}} \ldots\left|z_{k}\right|^{p_{k}+q_{k}} \leq L
$$

in

$$
0 \leq\left|z_{j}\right| \leq \frac{1}{4}
$$

Proof. If we put $z_{j}=\rho_{j} e^{i \varphi_{j}}, 0 \leq \varphi_{j}<2 \pi, \rho_{j}>0$, then

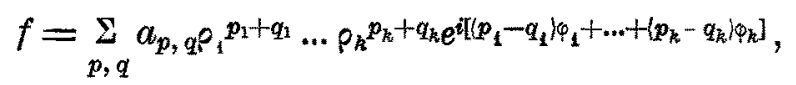

and if we put $p_{i}=q_{j}+r_{j}$ then this is

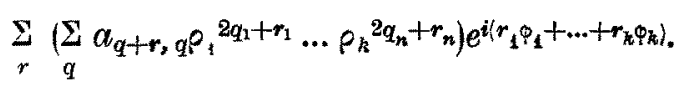

The latter expansion is a multiple FourIER series, and in such one each coefficient is maximized by the value of the function, so that, due to (34), we have

$$
\left|\sum_{q} a_{q+r, q} \rho_{1}^{2 q_{1}} \ldots \rho_{k}^{2 q_{k}}\right| \leq p_{1}^{-r_{1}} \ldots \rho_{k}-r_{k}
$$

for $0<\rho_{j}<2$. Therefore, for

$$
\frac{1}{2} \leq p_{j} \leq 2
$$

we have

$$
\left|\underset{q}{\mathbb{N}} a_{q+r, q P_{1}^{2}}^{2 q_{1}} \ldots \rho_{k}^{2 q_{k}}\right| \leq 2^{\left(r_{1}+\ldots+r_{k}\right)} .
$$


Next, there exist a number $L_{0}=L_{0}(k, l)$ such that if we have

$$
\left|q_{q_{j}=0}^{l} b_{q_{1} \ldots q_{k}} \rho_{1}^{2 q_{1}} \ldots \rho_{k}^{2 q_{k}}\right| \leq 1
$$

in $(36)$, then

$$
\underset{q_{j}=0}{l}\left|b_{q_{1}} \ldots q_{k}\right| \leq L_{0}
$$

In fact, if such a constant would not exist, there would be a sequence of systems of coefficients $\left\{c_{q_{1} \cdots q_{k}}^{\mu}\right\}, \mu=1, \ldots, 0 \leq q_{j} \leq l$, such that

$$
\underset{q_{j}=0}{l}\left|c_{q_{1}}^{\mu} \ldots q_{k}\right|=1
$$

and yet

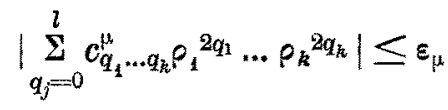

for $\rho_{1}, \ldots, \rho_{k}$ in (36), with $\varepsilon_{\mu} \rightarrow 0$. Due to the finiteness of $l$, we would then have coefficients $c_{q_{1} \ldots q_{k}}$ not all 0 , for which

$$
\sum_{q_{j}=0}^{l} c_{q_{1}} \ldots q_{k} \rho_{1}^{2 q_{1}} \ldots \rho_{k}^{2 q_{k}}=0
$$

for $\rho_{j}$ in (36), which for polynomials in $\rho_{j}$ is impossible.

Therefore, (37) implies

$$
\left|a_{q+r, q}\right| \leq 2^{\left(r_{1}+\ldots+r_{k}\right)} L_{0} .
$$

and therefore for $p_{j} \geq 0, q_{j} \geq 0$ we have

$$
\left|a_{p, q}\right| \leq 2^{p+\ldots p+h} L_{0}
$$

and hence

$$
\underset{p, q}{\Sigma}\left|a_{p, q}\right| 4^{-\left\{p_{1}+q_{1}+\ldots+p_{k}+q_{k}\right\rangle} \leq k^{l} L_{0}(k, l)=L_{1}(k, l),
$$

which proves the lemma.

4. Holomorphically-placed products af one-cycles.

A $k$-cycle $B_{k}$ in $D$ will be called a holomorphically-placed product if it is contained in some domain $D^{\prime}$ in $D$ such that a suitable one-one holomorphic mapping

$$
z^{j}=\varphi_{j}\left(w^{1}, \ldots, w^{k}\right)
$$

of $D^{\prime}$ transforms $B_{k}$ into a cycle which is rigidly-placed in the $w$-space. 
THeOREM 7. - If $f$ is continuous and if we have (21) for all holomorphi. cally-placed cycles, then $f$ is holomorphic.

Proof. First of all, by theorem $4, f$ can be represented by an expression (16) and uniquely so. Now, we claim that (16) must be independent of $z^{\alpha}$. In fact, if it would depend on $\bar{z}^{1}$, say, then the holomorphic transformation

$$
z^{1}=w^{1}+\left(w^{1}\right)^{k}, \quad z^{2}=w^{2}, \ldots, z^{k}=w^{k}
$$

would transform (16) into a polynomial

$$
b_{p_{1} \ldots p_{k}}(w)\left(\overline{w^{1}}\right)^{p_{1}} \ldots\left(\overline{w^{k}}\right)^{p_{k}}
$$

which is of a degree $z k$, but by the uniqueness of (16) in every system of holomorphic variables, this is impossible. Hence the theorem. 